\title{
A-KA Model: an Optimization of the Stock's Portofolio
}

\author{
Filippo Regina* \\ Mauro Gianfranco Bisceglia**
}

\begin{abstract}
The elaborate proposes a compact alternative methodology to the classical stocks portfolio optimization based on the normal distribution of the returns of the assets named Adaptable - Kurtosis Asymmetry model (A-KA). In the financial theory is well-known that odd-order moments of a distribution describe a particular performance characteristic; on the contrary, the even-order moments tell a precise sense of risk of a distribution of returns. If it is true that, in general terms, minimizing the variance also minimizes the volatility of portfolio return is also true that we should minimize the kurtosis to get away from unpleasant situations in case "Extreme" events occur, especially if negative. The idea behind this paper is to exploit the four moments of return's distributions, optimizing an alternative risk indicator to variance, such as the kurtosis of the final distribution of the portfolio, making constraints on distributive asymmetry, in a dynamic underlying logic.
\end{abstract}

Keywords: Asset Allocation; Portfolio construction; Stocks; Skewness; Kurtosis; Active Return; Rebalancing

\section{JEL Classification: G11}

\section{Introduction}

Recent works by T. Škrinjarić (2013) and H.-S. Ryoo (2007) has given the opportunity to develop a more "compact" model that aims to minimize the curtosis (preferably the one on the left tail) under the classic constraints of performance, balance and positivity, with the addition of the extra constraints on second and third order moments.

\footnotetext{
* Filippo Regina is M.Sc in Statistical Methods for Finance. E-mail address: filippo regina@live.it.

** Mauro Gianfranco Bisceglia is a corresponding author and the professor of Mathematical Methods of Economics and Actuarial and Financial Sciences - Dipartimento di Economia e Finanza, University of Bari "Aldo Moro". Postal address: Largo Abazia Santa Scolastica, 70124 Bari; Email address: maurogianfranco.bisceglia@uniba.it.
} 
Many iterations have led to the use of active strategies in order to make the model more dynamic: the first concerns the re-balancing of weight distribution and the second the targeted performance.

The problem of rebalancing and portfolio Turnover - which must necessarily be revised and must undergo periodic maintenance to maximize the profits created and to minimize any losses generated by sudden inversions of the market - has been discussed several times in the theory and many are the empirical works confirming the inevitable beneficial effect on Profits of that strategy.

In literature It has always been adopted as a goal (Markowitz, 1952; Konno and Yamazaki, 1991; Konno and Suzuki, 1995; Papahristodoulou and Dotzauer, 2004; Ryoo, 2007) a performance, which by definition is constant over time.

We wondered what the results would be, in terms of capital progressively cumulated over time, if you had chosen a value of $r^{*}$ that would adapt to the data available to each era of optimization.

The idea behind the consideration is that if we are in a Bull-type market, requiring the optimizer to calculate the weights of the individual securities in the portfolio in order to have an average $r \%$ return would not maximize the potentially desumible profits from the market. For this reason, one could try to set as objective a variable return fixed at each period of revision, according to an objective criterion: in this way we try to make the most of the market trend.

To make the best of the idea of maximum performance deducible from the market, maintaining the hypothesis of efficiency in the weak sense of the market and of homogeneous expectations of investors intuition is to build a distribution of expected average Adjusted-return for the risk of the investable universe's equity securities taken into account, calculated for each h-th time interval. At this point we use the concept of "quantile of order $\alpha$ " of the distribution to obtain the adjusted targeted return for the next sub-period, based on the one just observed. But that's not all.

Several times it was reiterated in the latest financial theory (G. Szegö, 2002) that odd-order moments of a distribution describe a particular performance characteristic; on the contrary, the even-order moments tell a precise sense of risk of a distribution of returns. If it is true that, in general terms, minimising the variance also minimises the volatility of portfolio return is also true that we should minimize the curtosis to get away from unpleasant situations in case "Extreme" events occur, especially if negative. In order to better appreciate this statement, which will be the mainstay of the A-KA model proposed subsequently, it necessitates a theoretical digression on the structure of the distributions of returns and on the relationship between risk and return. 


\section{The Sharpe ratio and the relationship between risk, Asymmetry and Kurtosis}

Once the estimators of average and variance of the linear returns of interests are defined, the estimator of the Sharpe index for the evaluation of an investment turns out to be:

$$
\widehat{S R}_{\iota}=\frac{\bar{r}_{l}-r_{f}}{s_{i}}
$$

where $r_{f}$ is the risk free rate, $\bar{r}_{i}$ is the average return and $s_{i}$ is the volatilityi.

Using the asymptotic statistical theory, through which there is the possibility of applying the central limit theorem to the estimators of $\mu$ and $\sigma 2$, we are capableto find the theoretical distribution of $\mathrm{s}$ and $\mathrm{r}$ in the hypothesis that $r_{t}$ is independent and equally distributed.

Assuming that the returns of the assets are i.i.d. and that they have finite average and variance, the $\mathrm{r}$ and $s^{2}$ estimators have an asymptotically normal distribution, due to the central limit theorem.

Specifically, for each i-th asset, the result is:

$$
\begin{aligned}
& \sqrt{T}\left(\bar{r}_{i}-\mu_{i}\right) \approx N\left(0 ; \sigma_{i}^{2}\right) \\
& \sqrt{T}\left(s_{i}^{2}-\sigma_{i}^{2}\right) \approx N\left(0 ; 2 \sigma_{i}^{4}\right)
\end{aligned}
$$

Where $\mathrm{T}$ is the Time range and $N($.$) is the Gaussian function.$

In this kind of model, the variance of the estimators aims to zero as the sample increases, showing how the estimation errors are reduced as the sample size increases.

Once the estimators of average and variance are found, the estimator of the Sharpe index can be found as a function of the estimators themselves, such as:

$$
\widehat{S R}_{i}=\frac{\bar{r}_{l}-r_{f}}{s_{i}}=g\left(\bar{r}_{l} ; s_{i}^{2}\right)
$$

which has, as asymptotic distribution ${ }^{\text {ii: }}$

$$
\sqrt{T}\left(\widehat{S R}_{\iota}-S R_{i}\right) \approx N\left(0 ; V_{i}\right)
$$

where it turns out that:

$$
V_{i}=\left(\frac{\partial g_{i}}{\partial \mu_{i}}\right) \sigma_{i}^{2}+\left(\frac{\partial g_{i}}{\partial \sigma_{i}^{2}}\right) 2 \sigma_{i}^{4}=1+\left(2 S R_{i}^{2}\right)^{-1}
$$

Of course, the results can be extended to a portfolio of assets, assuming that the variance of this portfolio is the weighted average of the variances of the assets with void covariances, because returns and risks are unrelated. Assuming that the return 
and risk of the different assets are independently and identically distributed (zero correlation) is an unrealistic hypothesis, therefore the model needs to be integrated, in order to take into account the non-independence and non-identical distribution existing between the matrix of Variances and Covariances.

DEFINITION 1: the modified Value at Risk, based on the Cornish-Fisher expansion of the $\alpha$ quantile of a non-Gaussian distribution, is defined as:

$$
M V a R_{\alpha}=\mu+z_{c f, \alpha} \sigma
$$

Where $z_{c f}$ is the Cornish-Fisher quantile $\mathrm{e}^{\mathrm{iii}}$, which is calculated as:

$$
z_{c f}=z_{\alpha}+\frac{1}{6}\left(z_{\alpha}^{2}-1\right) \gamma_{1}+\frac{1}{24}\left(z_{\alpha}^{3}-3 z_{\alpha}\right) \gamma_{2}-\frac{1}{36}\left(2 z_{\alpha}^{3}-5 z_{\alpha}\right) \gamma_{1}^{2}
$$

and $\gamma_{1}$ and $\gamma_{2}$ are the coefficients that measure asymmetry and kurtosis; $z_{\alpha}$ is the normal quantile.

Once we find the $z_{c f}$ quantity, the wording of the $\mathrm{i}$-th asset must be amended from $\sigma_{i}$ to $z_{c f} \sigma_{i}$.

The modified Value at Risk is used as an a risk measure which is alternative to the classic and standard deviation, in order to rectify the Sharpe ratios from an excess of kurtosis and asymmetry.

DEFINITION 2: The Modified Sharpe index is defined as:

$$
M S R_{\alpha, i}=-\frac{\bar{r}_{l}-r_{f}}{r_{f}-M V a R_{\alpha, i}}
$$

Generally, we find a more or less linear relationship between the Modified Sharpe index and the classic Sharpe index, but the empirical evidence has proved that strongly speculative assets have a highly asymmetric and leptokurtic distribution of returns and, in this case, the $\mathrm{MSR}_{\alpha}$ index becomes a key indicator in the choice of assets. 
Illustration 1: The relationship between S.R., M- S.R., S/K

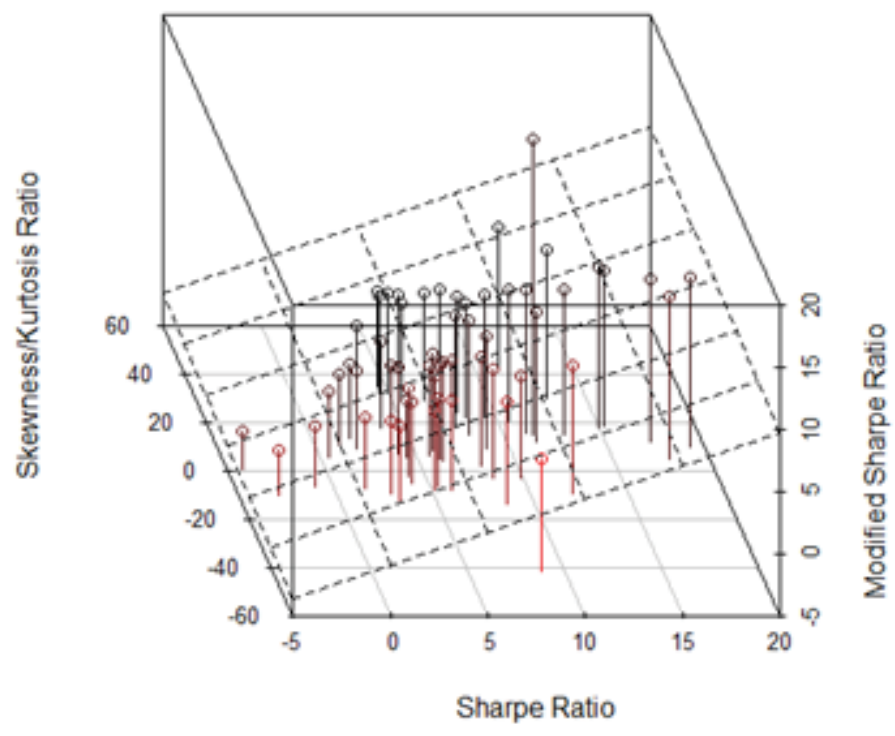

Source: Authors' elaboration of Italian Stocks Exchange data

In an essential empirical work, Cavenaile and Lejeune (2012) have proved how no confidence limits should ever be used below $95.84 \%$, in order to estimate the correctly modified Value at Risk and to obtain statistically valid results.

It is easy to perceive that, the considerable downward or upward correction of the MSR, compared to the classic Sharpe Ratio, is due to the Skewness ratio on Kurtosis.

With a fundamental theoretical contribution, Sura and Perron (2010) have shown that the relationship between Skewness and Kurtosis is described perfectly by a convex parabola, as it has been demonstrated in an empirical test in which the parabola is identified with an absolute minimum in $K=-20$ and $S=0.0$. 
Illustration 2: The relationship between Skewness and Kurtosis: empirical test

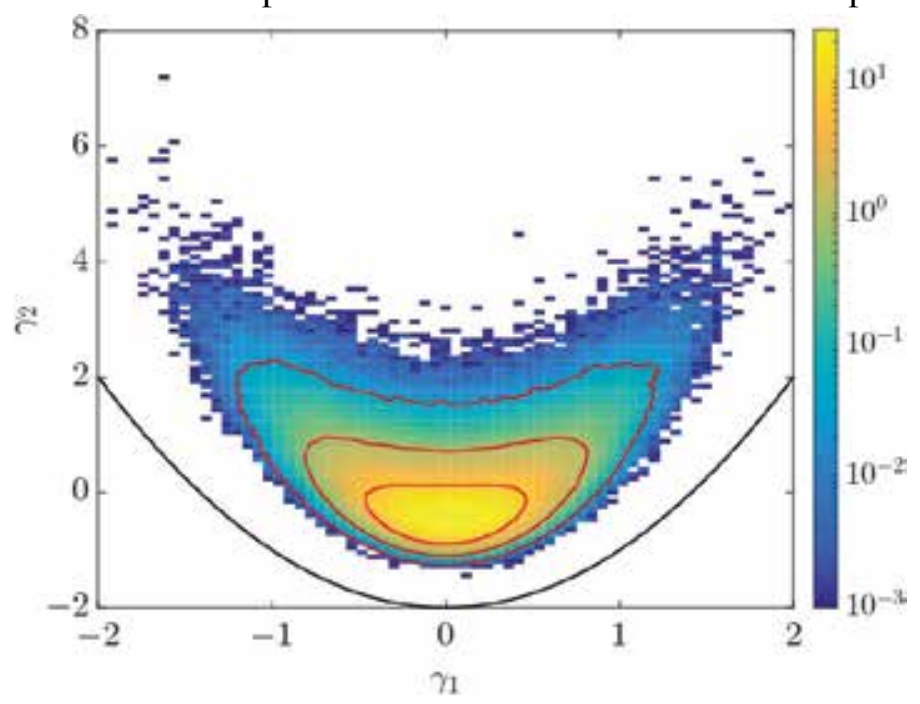

Source: Aggarwal, R., Rao R.P. \& Hiraki T. "Skewness and kurtosis in Japanese equity returns: empirical evidence”.

\section{A-KA model: Formalization and theory}

The adaptable model Kurtosis-Asymmetry arises from the need to minimize the excess of kurtosis in the distribution of portfolio returns, prioritizing stocks with positive performance asymmetry and adjusting the objective return to the ups and downs of the market.

In order to construct an algorithm which selects the adaptive objective performance, the following rule has been defined:

a) The average returns of the individual assets are calculated in the h-th last interval of time of $w$ extent available;

b) The quantile of order $\alpha$ is calculated with a simulation:

In this case, we must proceed as follows:

(1) The observations are ordered in an increasing order;

(2) The empirical quantile is calculated, which is the observation $q_{\alpha}$ such that $\alpha \%$ of the observations is lower than $q_{\alpha}$ and $(1-\alpha) \%$ is higher than $q_{\alpha}$.

c) If the return found is:

$$
\left\{r_{\left(q_{\alpha}\right)}\right\}_{h}>(M \cdot A \cdot R \cdot R \cdot)_{h}
$$

then we can fix as an objective efficiency $r(q)$ otherwise we fix as an objective (M.A.R.R.) hecided a priori (for example $0 \%$ in order to limit losses).

To simplify the model and to lose interest in the variance of the distribution of the returns of each $i$-th asset in the algorithm's iteration, we choose to use the C.A.P.M. 
system, in order to weight up linear returns through a weight system obtained by comparing the idiosyncratic risk to the total of idiosyncratic risks in the equidistributed portfolio. It should be noted that the idiosyncratic risk of the asset - which is the diversifiable risk - can be estimated from the equation:

$$
\sigma_{i}^{D I V}=\sigma_{i}-\sigma_{i}^{S I S T}=\sigma_{i}-\beta_{i} \sigma_{M}=\sigma_{i}-\frac{\sigma_{i}}{\sigma_{M}} \rho_{i, M} \sigma_{M}=\sigma_{i}-\sigma_{i} \rho_{i, M}
$$

Where $\sigma_{M}$ is the volatility of Market's return, $\rho_{i, M}$ is the correlation between Market's return and the Stock's return, and $\beta$ is the Beta of the stock estimated by C.A.P.M. ${ }^{\text {iv }}$

Therefore the i-th weight is described by the relation:

$$
w_{i}=\frac{\sigma_{i}^{D I V}}{\sum_{i=1}^{N} \sigma_{i}^{D I V}}
$$

In order to be able to estimate the weighting weights, we need $2 N$ estimations: in particular, the estimation of the beta of the individual assets is done through the regression equation of the market model:

$$
r^{*}=r_{f}+\beta_{i}\left(E\left[r_{M}\right]-r_{f}\right)
$$

The statement clearly arises from the fact that, according to Pat (2011), the beta of the assets in at least two subsequent financial years, are strongly correlated one another and are often stable over time, at least in a short term period.

Illustration 3: Strongly correlation of BETAs

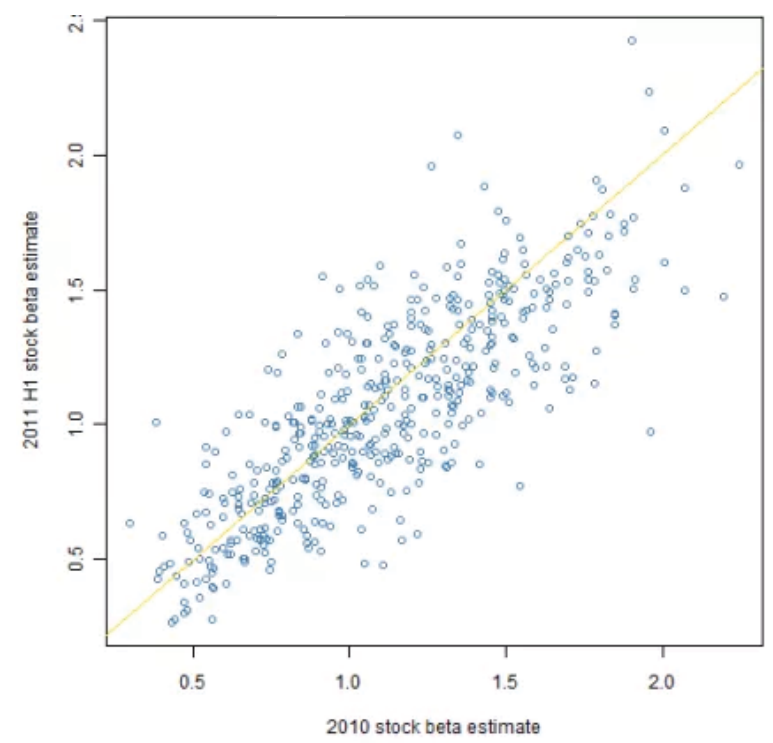

Source: Authors' elaboration of Italian Stocks Exchange data 
In 1996 Fama and French wrote a very interesting and revolutionary paper named: "Common Risk Factors in the returns on stocks and bonds". The two academics have introduced a principle which has made the fortune of many Hedge Funds in the 90s until 2008; in other words, they described and verified that the factors that conditioned and described the trend of certain assets compared to others were three, therefore not only the BETA but also the SMB and HML, or Small Minus Big and Small Minus Low (high compared to low).

Consequently, weighting can also be carried out, in a more elegant way, using factorial models that are nothing more than rankings, according to different indicators that identify quantitative characteristics of the assets that can be summarized and put in a ranking form.

The A-KA model therefore turns out to be:

$$
A-K A:\left\{\begin{array}{c}
\min \left[K\left(\sum_{j=1}^{n} r_{j h} x_{j h}\right)\right] \forall h=1,2, \ldots, H \\
\text { s.t. } \\
\sum_{t=1}^{T}\left(z_{t}^{+}\right)^{2}-k \sum_{t=1}^{T}\left(z_{t}^{-}\right)^{2} \geq 0 \\
\sum_{j=1}^{n} \bar{r}_{j h} x_{j h} \geq\left\{r_{q_{\alpha}}\right\}_{h-1} \\
\sum_{j=1}^{n} x_{j}=1 \\
\left\{x_{j}\right\}_{\forall j} \geq 0
\end{array}\right.
$$

where the objective function is the Kurtosis indicator, calculated on the vector of the portfolio returns proposed by J.J.A. Moors in $1988^{\mathrm{v}}$ and the constraints applied are those of positive asymmetry (according to H.-S. Ryoo), active return and canonical constraints of budget and of positive weights.

The basic concept is that Kurtosis can be interpreted as a measure of dispersion around the two limit values of the interval $m \pm s$, with a measure that results alternative to the parametric one, which is based on the fourth-order moment.

In formulas:

$$
K=\frac{\left(E_{3}-E_{1}\right)+\left(E_{7}-E_{5}\right)}{\left(E_{6}-E_{2}\right)}
$$

where:

I. $E_{1}$ is half of a quartile, which corresponds to the value occupying the 12.5 th percentile;

II. $E_{2}, E_{3}, \ldots, E_{7}$ are multiples of it.

In the below graph we can see how, the two terms of the numerator evaluate the concentration of data around $E_{6}$ and $E_{2}$, which correspond to the points $\mu \pm \sigma$. 
Graph 1: The Kurtosis, dispersion around the two limit values

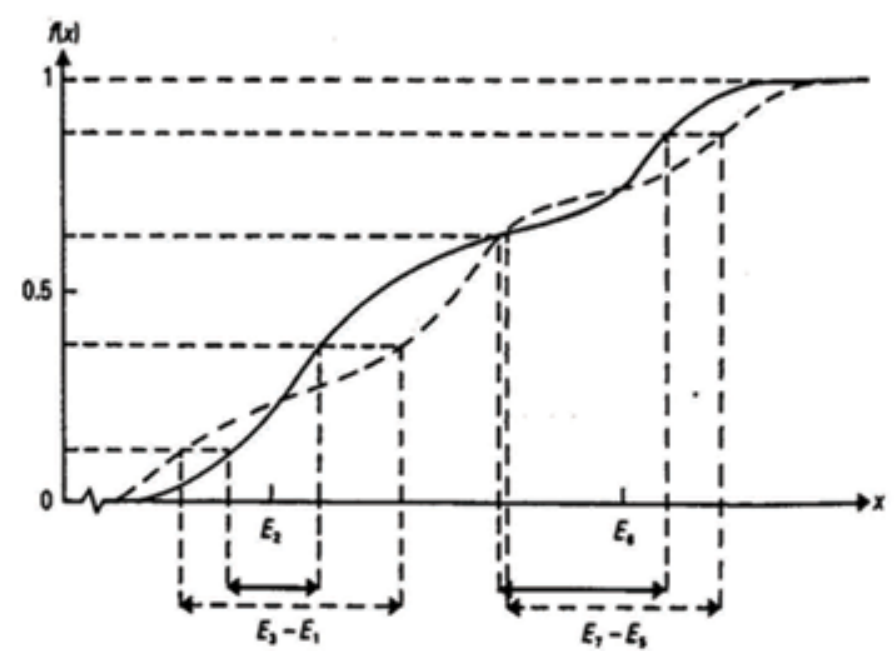

Source: Moors, J.J. (1988). A quantile alternative for kurtosis

The $K$ index is more robust than the parametric indexes, because it excludes the extreme values of the distribution. The denominator is a standardization constant that guarantees the invariance of $K$, regarding linear transformations.

If a distribution is symmetrical it results that:

$$
K=\frac{\left(E_{3}-E_{1}\right)+\left(E_{7}-E_{5}\right)}{\left(E_{6}-E_{2}\right)}=-\frac{\left(E_{3}-E_{1}\right)}{E_{2}}=+\frac{\left(E_{7}-E_{5}\right)}{E_{6}}
$$

To evaluate the functional form of p.d.f. it is necessary to make a comparison - in terms of Excess of Kurtosis - with the previously tabulated values of the standard Normal distribution, from which we can easily observe that:

$$
K_{N}=K_{N}^{+}=K_{N}^{-}= \pm 1,233
$$

The Positive Offset and Negative Offset functions ${ }^{\mathrm{vi}}$ should be defined as:

$$
\begin{aligned}
& \left(Z_{t}^{+}\right)=\left\{\begin{array}{r}
r_{t}-\bar{r}, r_{t}-\bar{r}>0 \\
0, r_{t}-\bar{r} \leq 0
\end{array}\right. \\
& \left(Z_{t}^{-}\right)=\left\{\begin{array}{r}
r_{t}-\bar{r}, r_{t}-\bar{r}<0 \\
0, r_{t}-\bar{r} \geq 0
\end{array}\right.
\end{aligned}
$$

Therefore the coefficient of asymmetry of H.S. Ryoo (2007), which is obtained by reporting only the positive and negative semi deviation, calculated as: 


$$
\begin{aligned}
& s^{+}=\sqrt{(T-1)^{-1} \sum_{t=1}^{T}\left(Z_{t}^{+}\right)^{2}} \\
& s^{-}=\sqrt{(T-1)^{-1} \sum_{t=1}^{T}\left(Z_{t}^{-}\right)^{2}}
\end{aligned}
$$

The idea behind the argument is simple: using the two reject functions, if the returns are symmetrical to the average, then the two subsets should have the same distribution $^{\text {vii. }}$ :

$$
\varphi(r)=\frac{\sum_{t=1}^{T}\left(z_{t}^{+}\right)^{2}}{\sum_{t=1}^{T}\left(z_{t}^{-}\right)^{2}}
$$

It is indicated with $h$ the rebalancing period, according to the " $w-f$ " strategy, which is appropriately calibrated and where the parameter a $\alpha$ indicates the $\%$ of the minor observations of $q_{a}$, the $f$ parameter indicates the frequency of the portfolio revision in a chosen period (daily, weekly, monthly, etc.) and the parameter $w$ indicates the width of the interval on which we need to calculate the average which are useful to the construction of the distributions. It must be specified that, in the empirical model, we must use the returns weighed with the procedure deriving from the C.A.P.M.

\section{An empirical test on the Italian stock market}

For the empirical simulation of the test, we have proceeded to build a portfolio based on the returns of the assets that construct the FTSE-MIB index of the Italian stock market, recorded between June 2016 and May 2017, with daily frequency.

We have previously carried out CUSUM-Q tests, in order to evaluate the stability of the Beta parameter for each asset; once we have verified it, we have proceeded to calculate the weight for the weighting of the asset return. 
Table 1: The coefficient for the weighting of the assets' returns

\begin{tabular}{|c|c|c|c|c|c|c|c|c|}
\hline ASSET & Beta & $\begin{array}{l}\text { Test } \\
\text { cusuMg }\end{array}$ & P-tralue & $\boldsymbol{\sigma}$ & $\boldsymbol{\sigma}$ stst. & $\boldsymbol{\sigma} \mathrm{Drv}$. & $\frac{\boldsymbol{\sigma}_{\text {SIST, }}}{\boldsymbol{\sigma}_{\text {DIV. }}}$ & W. \\
\hline A2A (EUR) & 0,69 & 0,868 & $68,49 \%$ & $1,26 \%$ & $0,69 \%$ & $0,57 \%$ & 1,20 & $3,24 \%$ \\
\hline ATLANTIA (EUR) & 0,71 & 1,111 & $31,03 \%$ & $1,09 \%$ & $0,70 \%$ & $0,39 \%$ & 1,82 & $2,19 \%$ \\
\hline Azimut (EUR) & 1,58 & 1,173 & $23,91 \%$ & $2,13 \%$ & $1,58 \%$ & $0,55 \%$ & 2,89 & $3,10 \%$ \\
\hline Banca Mediolanum (EUR) & 1,39 & 0,901 & $63,03 \%$ & $1,75 \%$ & $1,39 \%$ & $0,36 \%$ & 3,81 & $2,06 \%$ \\
\hline Banca Popolare di Sondrio (EUR) & 1,36 & 0,912 & $61,18 \%$ & $1,96 \%$ & $1,36 \%$ & $0,60 \%$ & 2,27 & $3,40 \%$ \\
\hline BPER BANCA (EUR) & 2,39 & 1,175 & $23,61 \%$ & $3,23 \%$ & $2,39 \%$ & $0,84 \%$ & 2,84 & $4,77 \%$ \\
\hline BUZZI UNICEM (EUR) & 1,18 & 1,063 & $37,35 \%$ & $1,71 \%$ & $1,18 \%$ & $0,53 \%$ & 2,22 & $3,02 \%$ \\
\hline CAMPARI (EUR) & 0,46 & 0,897 & $63,55 \%$ & $1,11 \%$ & $0,46 \%$ & $0,65 \%$ & 0,71 & $3,66 \%$ \\
\hline ENEL (EUR) & 0,72 & 0,753 & $85,69 \%$ & $1,13 \%$ & $0,72 \%$ & $0,41 \%$ & 1,74 & $2,34 \%$ \\
\hline ENI (EUR) & 0,88 & 0,720 & $89,65 \%$ & $1,27 \%$ & $0,88 \%$ & $0,40 \%$ & 2,20 & $2,25 \%$ \\
\hline Exgr (EUR) & 1,31 & 0,800 & $79,20 \%$ & $1,61 \%$ & $1,31 \%$ & $0,30 \%$ & 4,41 & $1,69 \%$ \\
\hline FERRARI NV (EUR) & 0,92 & 1,261 & $15,97 \%$ & $1,22 \%$ & $0,92 \%$ & $0,30 \%$ & 3,09 & $1,69 \%$ \\
\hline EinecaRaak (EUR) & 1,17 & 1,333 & $11,12 \%$ & $1,65 \%$ & $1,17 \%$ & $0,48 \%$ & 2,43 & $2,72 \%$ \\
\hline Generali Assicurazioni (EUR) & 1,58 & 1,007 & $45,64 \%$ & $2,07 \%$ & $1,58 \%$ & $0,49 \%$ & 3,24 & $2,76 \%$ \\
\hline INTESA SANPAOLO (EUR) & 1,95 & 1,081 & $34,93 \%$ & $2,43 \%$ & $1,95 \%$ & $0,48 \%$ & 4,07 & $2,71 \%$ \\
\hline LEONARDO (EUR) & 1,31 & 0,733 & $88,16 \%$ & $1,83 \%$ & $1,31 \%$ & $0,52 \%$ & 2,52 & $2,94 \%$ \\
\hline LUXOTTICA GROUP (EUR) & 0,63 & 0,803 & $78,75 \%$ & $1,38 \%$ & $0,63 \%$ & $0,75 \%$ & 0,84 & $4,25 \%$ \\
\hline MEDIASET (EUR) & 1,43 & 0,999 & $46,88 \%$ & $3,07 \%$ & $1,43 \%$ & $1,64 \%$ & 0,87 & $9,29 \%$ \\
\hline MEDIOBANCA (EUR) & 2,08 & 1,434 & $6,45 \%$ & $2,55 \%$ & $2,07 \%$ & $0,48 \%$ & 4,36 & $2,69 \%$ \\
\hline Moncler SDA (EUR) & 0,83 & 1,303 & $12,98 \%$ & $1,32 \%$ & $0,83 \%$ & $0,49 \%$ & 1,71 & $2,77 \%$ \\
\hline POSTE ITALIANE (EUR) & 0,86 & 1,288 & $13,96 \%$ & $1,15 \%$ & $0,86 \%$ & $0,29 \%$ & 2,92 & $1,66 \%$ \\
\hline Prysmian (EUR) & 1,15 & 1,199 & $21,25 \%$ & $1,43 \%$ & $1,15 \%$ & $0,28 \%$ & 4,09 & $1,60 \%$ \\
\hline $\begin{array}{l}\text { Recordati Industria Chimica e } \\
\text { Farmaceutica SAA (EUR) }\end{array}$ & 0,50 & 1,294 & $13,53 \%$ & $1,11 \%$ & $0,50 \%$ & $0,61 \%$ & 0,83 & $3,45 \%$ \\
\hline SAIPEM (EUR) & 1,30 & 1,256 & $16,53 \%$ & $2,33 \%$ & $1,30 \%$ & $1,03 \%$ & 1,27 & $5,82 \%$ \\
\hline $\begin{array}{l}\text { Salvatore Ferragamo Italia Spa } \\
\text { (EUR) }\end{array}$ & 0,9 & 0,537 & 9 & $\%$ & $\%$ & 0 & 1,35 & 3 \\
\hline Snam (EUR) & 0,55 & 1,139 & $27,63 \%$ & $1,11 \%$ & $0,54 \%$ & $0,57 \%$ & 0,96 & $3,21 \%$ \\
\hline ST MICROELECTRONICS (EUR) & 0,99 & 1,059 & $37,88 \%$ & $1,88 \%$ & $0,99 \%$ & $0,89 \%$ & 1,11 & $5,05 \%$ \\
\hline Telecom Italia (EUR) & 1,34 & 0,841 & $72,89 \%$ & $2,03 \%$ & $1,34 \%$ & $0,69 \%$ & 1,94 & $3,90 \%$ \\
\hline Tenaris (EUR) & 0,76 & 1,175 & $23,64 \%$ & $1,67 \%$ & $0,76 \%$ & $0,91 \%$ & 0,84 & $5,13 \%$ \\
\hline Terna (EUR) & 0,47 & 1,368 & $9,28 \%$ & $0,97 \%$ & $0,47 \%$ & $0,51 \%$ & 0,92 & $2,88 \%$ \\
\hline
\end{tabular}

Source: Authors' elaboration of Italian Stocks Exchange data

At this point, the weighted returns have been estimated and, for a better interpretation of the prices trend of the "correct" assets over time, they have been standardized to the value $O=100$ initially, and a logarithmic scale has been applied ${ }^{\text {viii }}$. 
The result is remarkable:

Illustration 4: Weighted standardized returns
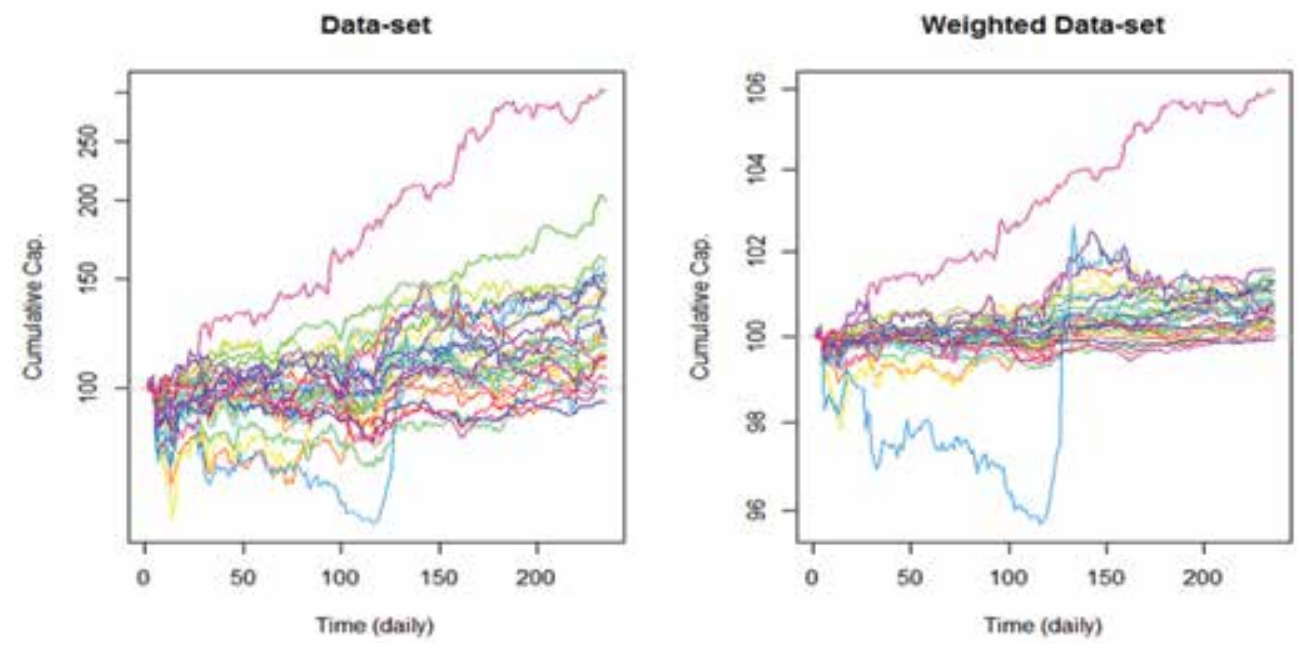

Source: Authors' elaboration of Italian Stocks Exchange data

By setting the $f-w$ parameters, a level of asymmetry required at 1.20 and a quantile at $90 \%$, the results obtained by the A-KA model, compared to those obtainable with the Markowitz model, with an objective efficiency set at $0.18 \%$ (retrospectively decided, on the basis of the arithmetic average return of the A-KA portfolio) and the FTSE-MIB are already graphically verifiable.

Illustration 5: A-KA vs Markowitz, Comparison with FTSE-MIB: daily cumulative perf. (re-scaled: 100)

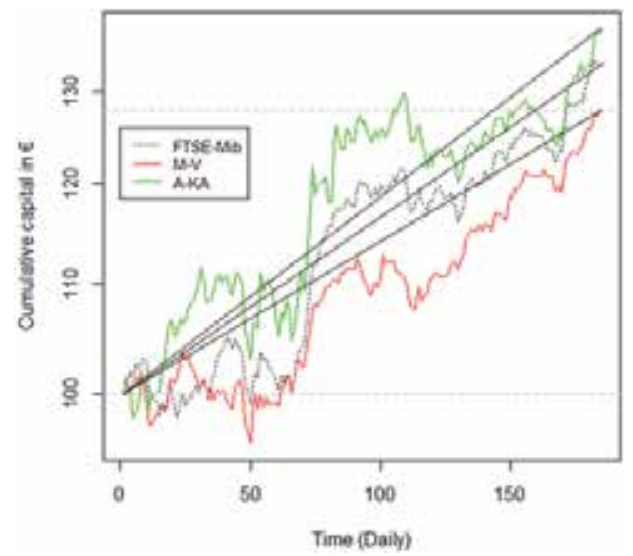

Source: Authors' elaboration of Italian Stocks Exchange data 
More accurately, the main calculated economic-financial statistics are summarized in the following table:

Table 2: The main economic-financial statistics

\begin{tabular}{|lrrr|}
\hline Ratio \& Index analysis & \multicolumn{1}{c}{$\boldsymbol{A}-\boldsymbol{K A}$} & \multicolumn{1}{c}{$\boldsymbol{M}-\boldsymbol{V}$} & \multicolumn{1}{c|}{$\boldsymbol{F T S E - M I B}$} \\
\hline Media troncata (10\%) & $0,13 \%$ & $0,12 \%$ & $0,14 \%$ \\
\hline Media & $0,18 \%$ & $0,14 \%$ & $0,16 \%$ \\
\hline Mediana & $0,13 \%$ & $0,05 \%$ & $0,16 \%$ \\
\hline Deviazione standard & $1,28 \%$ & $0,81 \%$ & $0,85 \%$ \\
\hline Curtosi & 2,81 & 1,66 & 1,15 \\
\hline Asimmetria & 0,81 & 0,46 & 0,26 \\
\hline Minimo & $-3,68 \%$ & $-2,37 \%$ & $-2,52 \%$ \\
\hline Massimo & $5,17 \%$ & $3,38 \%$ & $3,03 \%$ \\
\hline Skw/Krt Ratio & 0,29 & 0,27 & 0,23 \\
\hline Curtosi coda sx & $-0,98$ & $-1,33$ & $-1,19$ \\
\hline Excess Kurt. on Gaussian Distr. & $-0,26$ & 0,10 & $-0,05$ \\
\hline Curtosi coda dx & 1,43 & 1,99 & 0,94 \\
\hline Excess Kurt. on Gaussian Distr. & 0,20 & 0,75 & $-0,29$ \\
\hline Sharpe Ratio & $14,07 \%$ & $16,95 \%$ & $18,57 \%$ \\
\hline Normal Quantile & 1,96 & 1,96 & 1,96 \\
\hline VaR & $-2,33 \%$ & $-1,45 \%$ & $-1,51 \%$ \\
\hline Excess Return on VaR & $7,73 \%$ & $9,47 \%$ & $10,47 \%$ \\
\hline Cornish-Fisher Quantile & 2,44 & 2,26 & 2,15 \\
\hline Modified VaR & $-3,31 \%$ & $-1,96 \%$ & $-1,99 \%$ \\
\hline Exc. Ret. on MVaR $=$ Adj. SR & $5,45 \%$ & $6,98 \%$ & $7,94 \%$ \\
\hline Upperside Deviation & $0,91 \%$ & $0,57 \%$ & $0,58 \%$ \\
\hline Downside Deviation & $0,63 \%$ & $0,40 \%$ & $0,43 \%$ \\
\hline Variability Skewness & $144,84 \%$ & $139,76 \%$ & $134,18 \%$ \\
\hline
\end{tabular}

Source: Authors' elaboration of Italian Stocks Exchange data

The first quantitative reference results in a substantial difference between the average return obtained with the Media-Variance methodology and the one obtained with A-KA, which is however compensated enough by the difference between the standard deviations.

On the basis of the "classic" comparison parameters, summarized in the Sharpe-Ratio, we could conclude that the Media-Variance portfolio is better than the A-KA portfolio.

If we analyze in detail the indicators of functional form, we can easily observe that the classic Kurtosis index of returns distribution in the A-KA portfolio is higher than the one in the other two portfolios: on the other hand, however, the value of excess Kurtosis on the right tail is positive, even if lower than the one of the other two benchmark portfolios, but the symmetrical indicator that evaluates the excess on the left is negative. The negative sign of this indicator demonstrates that there is an underestimation of Kurtosis on the left tailin comparison with the mesokurtic distribution and therefore the distribution of the A-KA portfolio is "pulled to the right". 
This characteristic is immediately translated into a difference in the maximum return recorded in the value's accumulation period $(+5.17 \%$ against $+3.38 \%)$ with a minimum return threshold that is recorded with only one percentage point of difference, in comparison to the $\mathrm{M}-\mathrm{V}$ portfolio.

Illustration 6: Difference of the returns' distributions in the period of accumulation
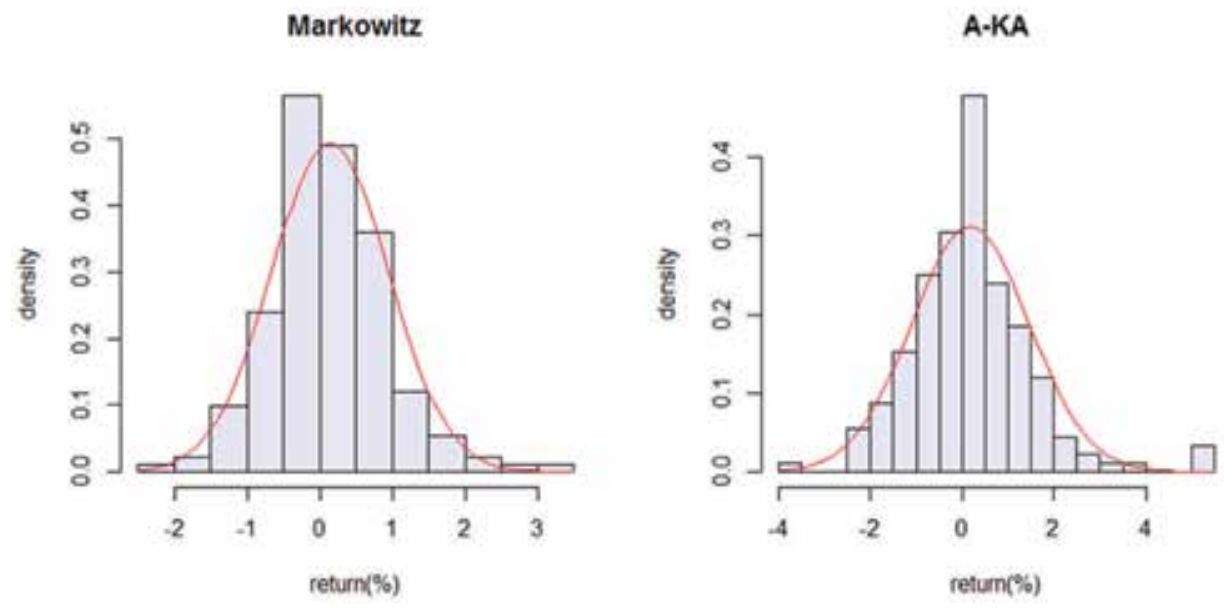

Source: Authors' elaboration of Italian Stocks Exchange data

On more accurate inspection, even if we deconstruct the risk in "Upperside" and "Downside" and if we calculate the Variability Skewness index ${ }^{\text {ix }}$, it turns out to be higher in the A-KA portfolio than in the Media-Variance portfolio.

In conclusion, we have constructed VaR models, to which we have submitted a back testing procedure, in order to understand whether the risk measurement models obtained were more or less able to correctly predict the maximum estimated loss in a given period.

Back testing procedures arise from the fact that the VaR is not observable in retrospect, therefore the classic statistics, such as the MSFE cannot be used to evaluate the average discrepancy between the theoretical model and the feasible model. For this reason we proceed to perform a test based on the expected value of the VaR.

Defining $I_{t}(\alpha)$ as the dichotomous variable "lower return of VaR":

$$
I_{t}(\alpha)= \begin{cases}1, & r_{t}<\operatorname{Var}_{t \mid t-1}(\alpha) \\ 0, & r_{t} \geq \operatorname{Var}_{t \mid t-1}(\alpha)\end{cases}
$$

According to P.F. Christoffersen (1998), the VaR prediction must satisfy two hypotheses of Unconditional Coverage (UCH) and Independence (INDH) ${ }^{\mathrm{x}}$. 
The UCH hypothesis states that:

I. If the expected value of the variable "return lower than VaR" is higher than alpha, the risk is underestimated;

II. If the expected value of the variable "return lower than VaR" is lower than alpha, then the risk is over-estimated;

The independence hypothesis tests the invalid hypothesis that the process which generates the variable "lower return of $\mathrm{VaR}$ " is i.i.d. against the alternative hypothesis that the process of failure is of Markov of the first order.

The combined hypothesis of Conditional Coverage can be tested through the statistics:

$$
L R_{C C}=L R_{U C}+L R_{I N D}
$$

which, under an invalid hypothesis, is distributed as a chi-square of 2 degrees of freedom.

For the two portfolios the calculated statistics are:

Table 3: The combined hypothesis of Conditional Coverage

\begin{tabular}{|c|c|c|c|}
\hline Hypotesis & Markowitz & A-KA & $\boldsymbol{\chi}^{\mathbf{2}}$ Value \\
\hline LRUC: Unconditional Coverage & 3,89 & 1,33 & 3,84 \\
\hline LRIND: Indipendence & 0,01 & 0,05 & 3,84 \\
\hline LRCC: ConditionalCoverage & 3,9 & 1,37 & 5,99 \\
\hline
\end{tabular}

Source: Authors' elaboration of Italian Stocks Exchange data

We can immediately observe that the VaR model, calculated on the Markowitz portfolio, does not satisfy the Unconditional Coverage hypothesis. The risk through the VaR model is, therefore, underestimated for this portfolio.

If we proceed with the calculation of the empirical alpha, by estimating the rolling VaR on 50 daily observations, it results that, in the $4.48 \%$ of the cases, the historical returns of the average-variance portfolio violate the historical VaR, against $1.49 \%$ of violations for the A-KA model. 
Illustration 7: Historical portfolio returns and historical VaR

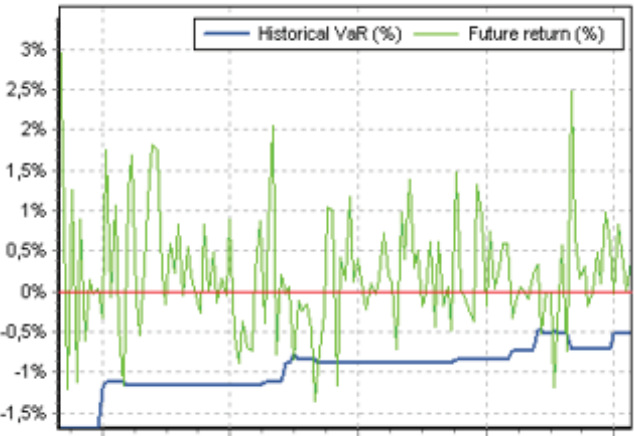

Future return of Markowitz portfolio

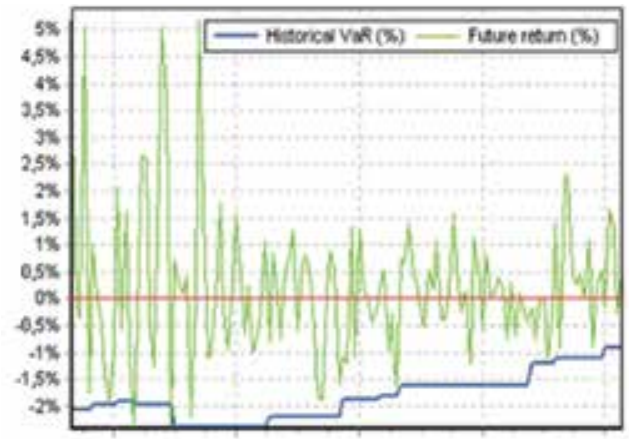

Future return of A-KA portfolio

Source: Authors' elaboration of Italian Stocks Exchange data

The quantitative results in both absolute and relative terms of the VaR model, which are calibrated on both portfolios, are summarized in the following table.

Table 4: The quantitative results of the VaR

\begin{tabular}{|c|c|c|c|c|}
\hline \multicolumn{5}{|c|}{ Value at Risk Results } \\
\hline & \multicolumn{2}{|c|}{ Markowitz } & \multicolumn{2}{|c|}{ A-KA } \\
\hline \multicolumn{5}{|c|}{ Absolute VaR } \\
\hline & Returns & Portfolio Value & Returns & Portfolio Value \\
\hline VaR & $-1,11 \%$ & $-1,42$ & $-1,81 \%$ & $-2,48$ \\
\hline CVaR- & $-1,44 \%$ & $-1,84$ & $-2,27 \%$ & $-3,11$ \\
\hline CVaR & $-1,46 \%$ & $-1,87$ & $-2,31 \%$ & $-3,16$ \\
\hline CVaR+ & $-1,47 \%$ & $-1,88$ & $-2,32 \%$ & $-3,18$ \\
\hline \multicolumn{5}{|c|}{ VaR relative to mean } \\
\hline & Returns & Portfolio Value & Returns & Portfolio Value \\
\hline VaR & $-1,25 \%$ & $-1,60$ & $-1,99 \%$ & $-2,73$ \\
\hline CVaR- & $-1,57 \%$ & $-2,01$ & $-2,45 \%$ & $-3,36$ \\
\hline CVaR & $-1,60 \%$ & $-2,05$ & $-2,49 \%$ & $-3,41$ \\
\hline $\mathrm{CVaR}+$ & $-1,61 \%$ & $-2,06$ & $-2,50 \%$ & $-3,43$ \\
\hline MVaR & $-1,96 \%$ & $-2,51$ & $-3,31 \%$ & $-4,54$ \\
\hline \multicolumn{5}{|c|}{ Base portfolio value to calculateVaR } \\
\hline Value & & 127,88 & & 137,26 \\
\hline Alpha & & $5,00 \%$ & & $5,00 \%$ \\
\hline Horizon & & 1,00 & & 1,00 \\
\hline
\end{tabular}

Source: Authors' elaboration of Italian Stocks Exchange data

Overall, we can state that the Modified VaR which modifies the quantile of distribution on the basis of the degree of asymmetry and kurtosis of the distribution of 
portfolio returns is more effective for the A-KA model, regarding risk containment, reducing the violation to just one case. The modified VaR calculation procedure, on the other hand, is completely useless in the Media-Variance model in terms of improving the number of violations.

\section{Conclusion and possible future developments}

The goal of this work is to deal more accurately the allocation of capital in a portfolio of risky assets.

The widespread media-variance approach, theorized for the first time by Markowitz in 1952, in fact, using the "minimal variance" solution in a problem which is deliberately multi-objective, considers equally "unpleasant" both the positive and negative deviations from the average.

This approach therefore starts from the construction of the Pareto frontier of efficient portfolios, and seeks the excellent Pareto "among the excellent" through the use of a utility function, which must however be a quadratic type and with precise parameters. For this reason, Markowitz himself had already revised the model in 1959, refining it through the use of the negative semi-function of the quadratic deviations from the average, as an objective function to be minimized.

Ryoo (2007) had included, in the methodology of construction of a risky portfolio, the perception of the modern investor about distributive asymmetry. Returning to the definitions of return and risk conferred by Giorgio Szegö, the doubt arose that, focusing on variance, albeit with due caution, is no guarantee of a $360^{\circ}$ focus on the risk characteristic of a financial asset since, in this analysis, the fourth distributive moment of the portfolio would be completely left aside.

So, using of a series of indicators and the market equilibrium model, a compact model, called adaptable Kurtosis-Asymmetry, has been constructed with the need to minimize the excess of kurtosis in the distribution of portfolio returns in comparison with the Normal distribution.

The methodological approach was the application of the model to a data-set, containing the daily quotations of the Italian market's shares, efficiently solving the problem with a hardware and software configuration, available to the average investor.

Further considerations were necessary after a direct statistical-financial comparison between the returns generated by the portfolio constructed with the A-KA method and those generated by the portfolio constructed with the classic method, considered a methodological benchmark during the entire length of the experiment.

For example, the A-KA portfolio has been classified as dominant, in terms of returns, risk and distribution form (asymmetrically positive and tending to platykurtic effect, in contrast to the currently accepted empirical financial theory), but it has been 
noted that the A-KA portfolio is also dominant in terms of diversification, which is much more pronounced than the one that the classic model offers.

The chosen approach is certainly interesting, while instead the authors of A-KA are already imagining how to refine the choice of goal performance in dynamic optics.

An hypothesis to the study for example, is to use the retrospective returns to construct " $n$ " simulations with Monte Carlo approach, to construct the volatility Ibbotson's cone and to select the objective return by positioning itself on a certain quantile.

Illustration 8: Simulated paths of the value of an asset using Monte Carlo

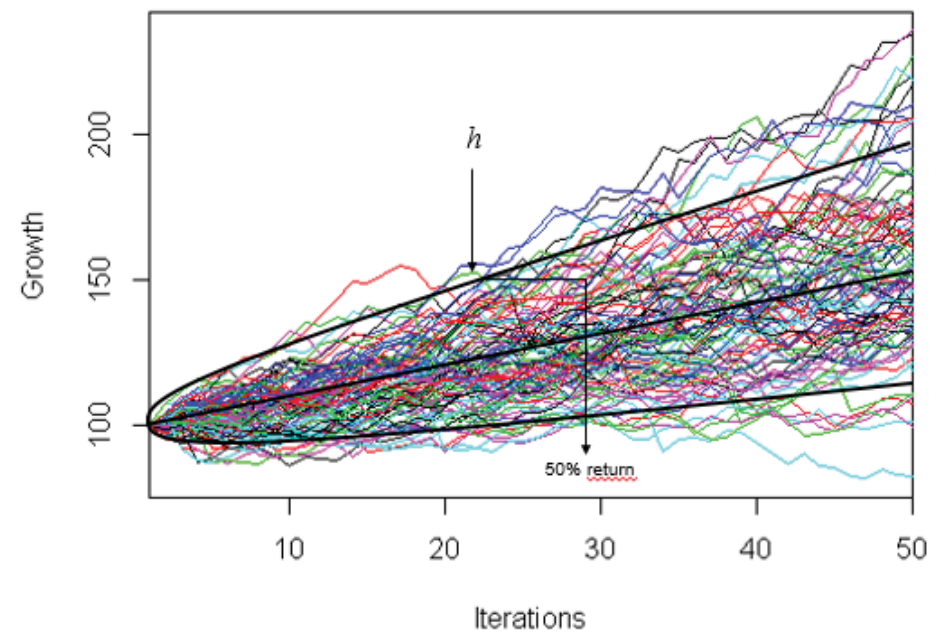

Source: Unreal elaboration of authors

This methodology certainly has the merit of working ex ante with a methodological approach prevision that clearly improves the choice of expected performance making it even more precise and aware.

\section{NOTES}

${ }^{\text {i }}$ Sharpe, W. (1994). “The Sharpe Ratio.” Journal of Portfolio Management, vol. 21, no. 1 (Fall):49-58. ii Lo Andrew W. (2002); "The Statistics of SharpeRatios", Hedge funds

iii Jaschke S.R. (2002) The Cornish-Fisher-Expansion in the Context of Delta-Gamma-Normal Approximations. The Journal of Risk, 4 (4), 33 - 52

${ }^{\text {iv }}$ Fama, E. and MacBeth, J., (1973), “Risk, Return and Equilibrium: Empirical Tests,” Journal of Political Economy 81, 115-146 
" Moors, J.J. (1988); “A quantile alternative for kurtosis”, The Statistician, 37, 25-32.

${ }^{v i}$ Markowitz H.M., Todd P., Xu G., Yamane Y. (1993); Computation of mean-semivariance efficient sets by the critical line algorithm; Annals of Operations Research 45: 307-317.

vii Ryoo H.S. (2007); A compact mean-variance-skewness model for large scale portfolio optimization and itsapplication to the NYSE market; The Journal of the OperationalResearch Society 58: 505-515.

viii The logarithmic scale is a graphical representation of positive real numbers. We associate to the points of a half-line the positive real numbers greater than zero as follows: if $\mathrm{O}$ is the origin of the half-line and $\mathrm{P}$ is the point that must represent the $\mathrm{x}>0$ number, the length of the OP segment which, from now on, we will indicate with $\lambda(O P)$ is proportional to $\log _{10}(\mathrm{x})$. This geometric representation of positive real numbers is called logarithmic scale. The $\mathrm{O}$ point will clearly represent the number 1 ; by indicating with $\mathrm{x}_{\mathrm{o}}$ the number which is represented by $\mathrm{O}$, we have indeed

$\lambda(O O)=0 \Leftrightarrow \log _{10}\left(x_{0}\right)=0 \Leftrightarrow x_{0}=10^{0}=1$

ix We would like to remind that Upperside deviation, or positive variability, is certainly well accepted by the modern investor contrary to Downside deviation, or negative variability. It should also be remembered that the Variability Skewness coefficient is nothing but the relationship between "good risk" and "bad risk".

× Christoffersen P. e Pelletier D. (2004); "Backtesting Value-at-Risk: A Duration-BasedApproach"; Journal of Financial Econometrics, 84-108.

\section{REFERENCES}

Aggarwal, R., Rao R.P. \& Hiraki T. (1989). Skewness and kurtosis in Japanese equity returns: empirical evidence. The Journal of Financial Research. 12(3), 253-260. DOI: 10.1111/j.1475-6803.1989. tb00518.x.

Artzner, P., Delbaen F., Eber J.-M. \& Heath D. (1999). Coherent Measures of Risk. Mathematical Finance. 9(3), 203-228. DOI: 10.1111/1467-9965.00068. Retrieved February 3, 2011.

Cantelli, F. P. (1933). Sulla determinazione empirica delle leggi di probabilità. Giornale Istituto Italiano Attuari. 4, 221-424.

Cavenaile, L. \& Lejenne T. (2012). A Note on the Use of the Modified Value-at-Risk. Journal of Alternative Investments, Forthcoming. 14(4), 79-83. DOI: 10.3905/jai.2012.14.4.079. Last revised 3 feb. 2016.

Chong, J. \& Phillips M. G. (2011). Measuring risk for cost of capital: The downside beta approach. Journal of Corporate Treasury Management. 4(4), 344-352. Retrieved 26 June 2013.

Christoffersen, P. \& Pelletier D. (2004). Backtesting Value-at-Risk: A Duration-Based Approach. Journal of Financial Econometrics. 2(1), 84-108. DOI: 10.1093/jjfinec/nbh004.

Fama, E.F. \& French K.R. (1996). Multifactor Explanations of Asset Pricing Anomalies. Journal of Finance. 51(1), 55-84. DOI: 10.1111/j.1540-6261.1996.tb05202.x.

Fama, E.F. \& MacBeth, J.D. (1973). Risk, Return and Equilibrium: Empirical Tests. Journal of Political Economy. 81(3), 607-636. DOI: 10.1086/260061.

Glivenko, V. (1933). Sulla determinazione empirica della legge di probabilità. Giornale Istituto Italiano Attuari. 4, 92-99.

Glivenko, V., (1933): Sulla determinazione empirica della legge di probabilità, Giornale Istituto Italiano Attuari, 4, 92-99.

Jaschke, S.R. (2002). The Cornish-Fisher-Expansion in the Context of Delta-Gamma-Normal Approximations. The Journal of Risk. 4(4), 33-52. DOI: 10.21314/JOR.2002.068. 
Konno, H. \& Yamazaki H. (1991). Mean-absolute deviation portfolio optimization model and its application to Tokyo Stock Market. Management Science. 37(5), 519-531. DOI: 10.1287/ mnsc.37.5.519.

Konno, H. \& Suzuki K.-I. (1995). A mean-variance-skewness portfolio optimization model. Journal of the Operations Research Society of Japan. 38 (2), 173-187. DOI: 10.15807/jorsj.38.173

Lo Andrew, W. (2002). The Statistics of Sharpe Ratios. Financial Analysts Journal. 58(4), 36-52. DOI: 10.2469/faj.v58.n4.2453.

Maghrebi, N. (1992). On skewness preference and persistence hypothesis. Japan Financial Review. 15, 17-35. DOI: R000000004-I3472569-00

Markowitz, H.M. (1952). Portfolio selection. Journal of Finance. 7(1), 77-91. DOI: 10.1111/j.15406261.1952.tb01525.x

Markowitz, H.M. (1991). Portfolio Selection (2nd edition). Malden Massachusetts: Blackwell.

Markowitz, H.M., Todd P., Xu G. \& Yamane Y. (1993). Computation of mean-semivariance efficient sets by the critical line algorithm. Annals of Operations Research. 45(1), 307-317. DOI: 10.1007/BF02282055.

Moors, J.J. (1988). A quantile alternative for kurtosis. Journal of the Royal Statistical Society. Series D The Statistician. 37(1), 25-32. DOI: 10.2307/2348376.

Papahristodoulou, C. \& Dotzauer E. (2004). Optimal Portfolios Using Linear Programming Models. The Journal of the Operational Research Society. 55(11), 1169-1177. DOI: 10.1057/palgrave. jors.2601765.

Roll, R. (1977). A Critique of the Asset's Pricing Theory's Tests: Part I. Journal of Financial Economics. 4(2), 129-176. DOI: 10.1016/0304-405X(77)90009-5.

Ross, S.A. (1976). The arbitrage theory of capital asset pricing. Journal of Economic Theory. 13(3), 341-360. DOI: 10.1016/0022-0531(76)90046-6.

Ryoo, H.S. (2007). A compact mean-variance-skewness model for large scale portfolio optimization and its application to the NYSE market. Journal of the Operational Research Society. 58(4), 505-515. DOI: 10.1057/palgrave.jors.2602168.

Sharpe, W.F. (1964). Capital Asset Prices: A Theory of Market Equilibrium under Conditions of Risk. The Journal of Finance. 19(3), 425-442. DOI: 10.2307/2977928.

Škrinjarić, T. (2013). Portfolio Selection with Higher Moments and Application on Zagreb Stock Exchange. Zagreb International Review of Economics \& Business. 16(1), 65-78. DOI: https:// hrcak.srce.hr/102022.

Sortino, F. A. \& Stephen S. (2001). Managing Downside Risk in Financial Markets: Theory, Practice and Implementation. Butterworth-Heinemanne: Elsevier Ltd.

Sura, P. \& Perron M. (2010). Extreme Events and the General Circulation: Observations and Stochastic Model Dynamics. American Meteorological Society. Journals online https://doi. org/10.1175/2010JAS3369.1.

Varadhan, R. (2012). Package "ALABAMA"; R Reference Manual; Johns Hopkins University School of Medicine, Baltimore, Maryland. 